\title{
Greater admissions, mortality and cost of heart failure in adults with congenital heart disease
}

\author{
Danielle S Burstein (1) , ${ }^{1}$ Joseph W Rossano, ${ }^{1}$ Heather Griffis, ${ }^{2}$ Xuemei Zhang, ${ }^{2}$ \\ Rachel Fowler, ${ }^{3}$ Benjamin Frischertz, ${ }^{3}$ Yuli Y Kim 이 ${ }^{1,4}$ JoAnn Lindenfield, ${ }^{3}$ \\ Jeremy A Mazurek, ${ }^{4}$ Jonathan B Edelson, ${ }^{1}$ Jonathan N Menachem ${ }^{3}$
}

- Additional material is published online only. To view, please visit the journal online (http://dx.doi.org/10.1136/ heartjnl-2020-318246).

${ }^{1}$ Division of Pediatric Cardiology, Children's Hospital of Philadelphia, Philadelphia, Pennsylvania, USA

${ }^{2}$ Healthcare Analytics Unit, Children's Hospital of Philadelphia, Philadelphia, Pennsylvania, USA

${ }^{3}$ Division of Cardiology, Vanderbilt University Medical Center, Nashville, Tennessee, USA

${ }^{4}$ Division of Cardiovascular Medicine, Hospital of the University of Pennsylvania, Philadelphia, Pennsylvania, USA

Correspondence to

Dr Danielle S Burstein, Division of Pediatric Cardiology,

Childrens Hospital of

Philadelphia, Philadelphia, PA 19103, USA;

bursteind@email.chop.edu

Received 15 September 2020 Revised 4 December 2020 Accepted 8 December 2020 Published Online First 23 December 2020

\section{Linked}

- http://dx.doi.org/10.1136/ heartjnl-2020-318757

Check for updates

(C) Author(s) (or their employer(s)) 2021. No commercial re-use. See rights and permissions. Published by BMJ.

To cite: Burstein DS Rossano JW, Griffis H, et al. Heart 2021;107:807-813.

\begin{abstract}
Objective Limited contemporary data exist regarding outcomes and resource use among adults with congenital heart disease and heart failure (ACHD-HF). This study compared outcomes, emergency department (ED) and hospital resource use, and advanced heart failure (HF) therapies in ACHD-HF versus non-ACHD with HF (HF-non-ACHD)
\end{abstract}

Methods The Nationwide Emergency Department Sample and Nationwide Inpatient Sample were used to analyse outcomes and resource use among ACHD-HF ED visits and hospitalisations from 2006 to 2016. ACHD-HF was stratified by single-ventricle (SV) and two-ventricle (2V) disease.

Results A total of 76557 ACHD-HF visits (3.6\% SV physiology) and 31137414 HF-non-ACHD visits were analysed. ACHD-HFs were younger (SV 33 years (IQR 25-44), 2V 62 years (IQR 45-76); HF-non-ACHD 74 years (IQR 63-83); $p<0.001)$. ACHD-HFs had higher ED admissions ( $78 \%$ vs $70 \%, p<0.001)$, longer hospital length of stay ( 5 days (IQR 2-8) vs 4 days (IQR 2-7), $\mathrm{p}<0.001)$ and greater hospital costs ( $\$ 49 \mathrm{~K}$ (IQR $2 \mathrm{~K}-121 \mathrm{~K})$ vs $\$ 32 \mathrm{~K}(17 \mathrm{~K}-66 \mathrm{~K}), \mathrm{p}<0.001)$. Mortality was significantly higher among ACHD-HFs with SV physiology (6.6\%; OR 1.6, 95\% CI 1.1 to 2.3 ) or $2 \mathrm{~V}$ physiology (6.3\%; OR $1.4,95 \% \mathrm{Cl} 1.3$ to 1.5$)$ versus HF-non-ACHD (5.5\%). ACHF-HF hospitalisations increased more $46 \%$ vs $6 \%$ HF-non-ACHD) over a 10 -year period, but the proportion receiving ventricular assist device (VAD) (ACHD-HF - 2\% vs HF-non-ACHD 294\%) or transplant (ACHD-HF -37\% vs HF-non-ACHD 73\%) decreased.

Conclusion ACHD-HFs have significant ED and hospital resource use that has increased over the past 10 years. However, advanced HF therapies (VAD and transplantation) are less commonly used compared with those without adult congenital heart disease.

\section{INTRODUCTION}

As surgical and medical care for congenital heart disease (CHD) has advanced, survival has improved dramatically, and today nearly $90 \%$ of infants with CHD survive to adulthood. ${ }^{1-3}$ This improved survival is accompanied by a rapidly growing population of adults with $\mathrm{CHD}-1.4$ million adults with CHD currently living in the USA. In fact, the adult congenital heart disease (ACHD) population now numerically exceeds the paediatric CHD population; additionally, it is growing by over 50000 patients per year. ${ }^{4}$ Simultaneously, there has been a shift in ACHD care towards a focus on longterm morbidities, including arrhythmias, pulmonary hypertension and heart failure (HF). HF is a highly morbid complication that results from prior surgical interventions, residual lesions, pressure or volume overload states, coronary anomalies and arrhythmias. $^{5} 6$ Adverse myocardial remodelling and neurohormonal activation further contribute to ventricular dysfunction, causing $\mathrm{HF}$ to progress in severity over time. While the patients most at risk of ACHD-related HF (adults with congenital heart disease and heart failure (ACHD-HF)) include those with single-ventricle (SV) physiology, tetralogy of Fallot and transposition of the great arteries, more recent data also demonstrate even adults with lower-complexity CHD have substantial cardiovascular morbidity and risk of $\mathrm{HF}^{78}$

Among patients with ACHD, HF is the leading cause of mortality, comprising $45 \%$ of cardiovascular deaths. ${ }^{9} 10$ Patients with ACHD who are hospitalised for an HF exacerbation have significantly higher mortality compared with patients with ACHD hospitalised for other reasons. ${ }^{11-14} \mathrm{HF}$ presentation comprises a significant proportion of hospital admissions: $2.5 \%$ of patients with ACHD are hospitalised annually, and of those, $20 \%$ are hospitalised for HF. ${ }^{11}$ As compared with adults without CHD who are hospitalised for HF, patients who are ACHD-HFs are significantly younger and have fewer overall comorbidities. ${ }^{15}$

Burchill et al described hospital resource use in the ACHD-HF population between 1998 and 2011 and demonstrated dramatic increases in ACHD-related HF hospitalisations and procedures (including intra-aortic balloon pump and mechanical circulatory support). ${ }^{15}$ However, an Interagency Registry for Mechanically Assisted Circulatory Support report demonstrated increasing use of ventricular assist device (VAD) in this population over recent years that were not captured in Burchill's study period. ${ }^{16}$ Additionally, data regarding emergency department (ED) use in this population are limited. Thus, contemporary data regarding outcomes and resource use among ACHD with HF are needed. We aimed to study differences in resource use among ACHD-HFs and non-ACHD with HF (HF-non-ACHDs), and we hypothesise that ACHD-HFs have worse outcomes but greater ED and hospital resource use and costs compared with adults without CHD. We will also evaluate trends in resource use for ACHD-HF care 
over a recent 10-year period and determine whether disparities exist in the use of advanced HF therapies in ACHD-HFs compared with adults without CHD.

\section{METHODS}

\section{Data source}

Data regarding hospital care were analysed using data from the Nationwide Inpatient Sample (NIS), the largest publicly available inpatient care database in the USA. ${ }^{17}$ The NIS also contains a stratified sample of $20 \%$ of US academic and non-academic hospitals. Data regarding ED care were obtained using discharge data from the Nationwide Emergency Department Sample (NEDS), the largest publicly available all-payer ED database in the USA. NEDS contains a $20 \%$ stratified sample of ED visits throughout the USA with data collected from the Healthcare Cost and Utilisation Project (HCUP) within the Agency for Healthcare Research and Quality ${ }^{18} 19$ NEDS data are provided by the HCUP State Inpatient Databases and the State Emergency Department Databases and captures discharge information after ED visits. Both NEDS and NIS provide appropriate weights to obtain weighted national estimates.

\section{Study population}

Data from the NEDS and NIS database from 2006 to 2016 were analysed. The unit of analysis is based on encounter level during ED or hospital visit (not patient level). Patients were included if they were $>18$ years of age, had an International Classification of Disease, 9th Revision (ICD-9) or International Classification of Disease, 10th Revision (ICD-10), diagnosis code for HF or cardiogenic shock (online supplemental table 1). Patients with ACHD were identified and categorised as either SV or twoventricle (2V) ACHD based on ICD-9 or ICD-10 diagnosis codes (online supplemental table 1). Diagnosis codes with lower specificity for ACHD, including atrial septal defect, bicuspid aortic valve, aortic stenosis and unspecified congenital anomalies, were excluded, as has been done previously. ${ }^{15}$ Diagnosis code for pregnancy, other connective tissue disease, heart transplant and heart-lung transplant were also excluded. Comorbidities were identified using diagnosis codes and included arrhythmia, pulmonary hypertension, hypertension, diabetes, chronic kidney disease, obesity, smoking, chronic obstructive pulmonary disease and coronary artery disease. Chronic complex conditions were defined as medical conditions that would reasonably be expected to last $>12$ months and involve either several different organ systems or one organ system severely affected enough to require specialty care and hospitalisation in a tertiary care centre; this was based on a previously published classification scheme. ${ }^{20}$ Procedures were captured using ICD-9 and ICD-10 codes (online supplemental table 1) and included VAD, extracorporeal membrane oxygenation (ECMO), heart or heart-lung transplantation, intra-aortic balloon pump (IABP), internal cardiac defibrillator (ICD) and Swann/central venous catheter/right heart catheterisation.

\section{Patient and public involvement}

Patients were not involved in this research.

\section{Outcomes of interest}

Study outcomes included mortality, disposition and resource use across ED visits and hospitalisations in both ACHD-HFs and HF-non-ACHDs. Regarding ED-related outcomes, we examined the frequency and trends of ED visits during the study period, ED visits disposition (admission, discharged or died) and ED-related costs across cohorts. We then evaluated number and trend in HF hospitalisations during the study period, hospital mortality, hospital length of stay (LOS), hospitalisation costs and rates of invasive procedures, including advanced HF therapies (VAD, ECMO, heart or heart-lung transplantation, IABP, ICD implantation and central venous catheter/right heart catheterisation).

\section{Statistical analysis}

Demographic and clinical data are reported as number with per cent or median value with IQR, as appropriate. Comparison of characteristics between patients with HF with ACHD and those without ACHD were assessed with $\chi^{2}$ tests or linear regressions that take account of the stratified cluster sample. Trend analyses using Cochran-Armitage tests for rates were conducted. ED and hospital costs were inflated to 2016 US dollars using the medical care and hospitalisation component of the personal consumption expenditure index. ${ }^{21}$ To test the association of patient-level or centre-level characteristics with outcomes, including mortality and resource use, multivariable logistic regression models or multiple linear regressions were fitted with appropriate covariates, adjusting for age, gender, insurance type, hospital affiliation (academic vs non-academic), year and comorbidities. Log transformation for the continuous outcome variables, hospital LOS, hospital cost and ED cost was used to achieve the approximation of normal distribution. All national estimates, 95\% CIs, IQRs, trend tests, logistic regressions and linear regressions were conducted by using SAS survey procedures, as recommended by HCUP, to compute accurate variances. ${ }^{17}$ All analyses accounted for the HCUP's complex survey designs using appropriate survey weights. Due to the 2012 NIS database redesign, NIS trend weights were used for NIS data before 2012. All data management and statistical analysis were performed using SAS V.9.4. A two-sided $\mathrm{p}$ value of $<0.05$ was used as the threshold for statistical significance.

\section{RESULTS}

\section{Patient cohort}

The study cohort is shown in figure 1 and patient demographics are shown in table 1. A total of 84963 ED visits (based on NEDS database) occurred in ACHD-HFs from 2006 to 2016, with 2252 (3\%) patients who were SV-ACHD-HFs; during the same period, there were 51970415 HF-non-ACHD ED visits. As shown in table 1, based on NIS data, patients who were ACHD-HFs were younger compared with HF-non-ACHDs (median age 61 (IQR 44-78) vs 74 (IQR 62-83) years, p value <0.001) (figure 2); SV-ACHD-HFs were the youngest cohort (median age 33 (IQR 25-43) years, $p$ value $<0.001)$. ACHD-HFs were more often treated in teaching hospitals, with $73 \%$ of patients who were SV-ACHD-HFs and 54\% of patients who were 2V-ACHD-HFs cared for in a teaching hospital compared with $45 \%$ of those with HF-non-ACHD ( $p$ value $<0.001$ ). Insurance patterns differed across groups, with patients who were SV-ACHD-HFs more often being insured by Medicaid or private insurance, while HF-non-ACHDs were more often on Medicare ( $p$ value $<0.001$ ). ACHD-HFs had a higher incidence of arrhythmias and pulmonary hypertension but a lower incidence of acquired comorbidities, such as diabetes, chronic kidney disease, obesity and coronary artery disease.

\section{ACHD-related HF care in EDs}

In patients with $\mathrm{HF}$ who presented to the ED, ACHD-HFs had higher rates of hospital admission compared with HF-nonACHDs (SV-ACHD-HFs: OR 1.51, 95\% CI 1.18 to 1.94, 

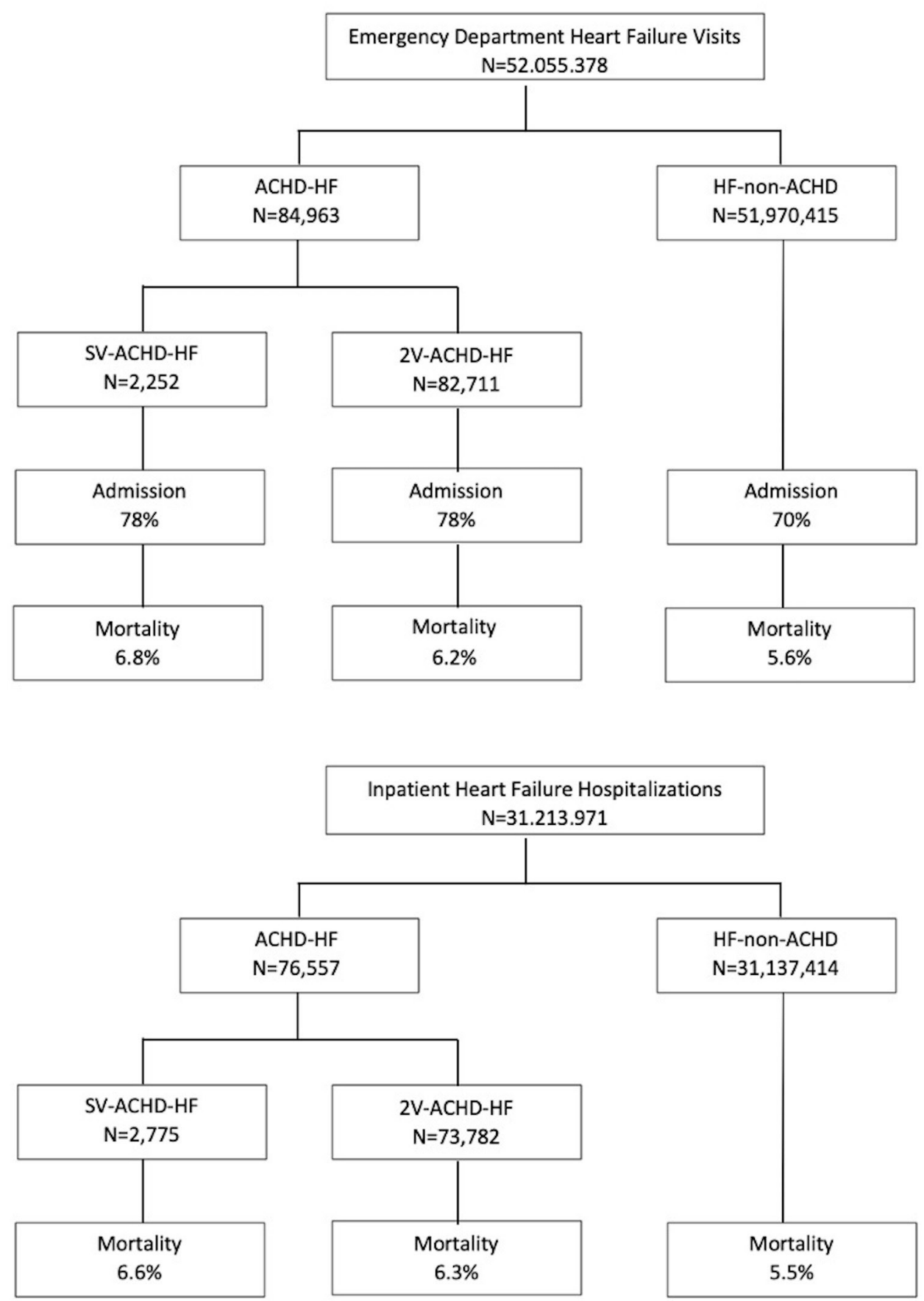

Figure 1 Study cohort of HF visits to the emergency department (top: based on NEDS database) and inpatient (bottom: based on NIS database) from 2006 to 2016. 2V, two-ventricle; ACHD-HF, adults with congenital heart disease and heart failure; HF, heart failure; NEDS, Nationwide Emergency Department Sample; NIS, Nationwide Inpatient Sample; SV, single-ventricle.

p value $<0.001 ; 2 \mathrm{~V}$-ACHD-HFs: OR 1.48 , 95\% CI 1.39 to 1.56 , $\mathrm{p}$ value $<0.001$; online supplemental table 2 ). Although mortality in the ED was low across all groups, SV-ACHD-HFs had twice the ED mortality compared with others $(0.6 \%$ vs 2V-ACHD-HF mortality $0.3 \%$ vs HF-non-ACHD mortality $0.3 \%$, p value $<0.001)$. Median ED-related hospital costs were similar for ACHD-HFs (\$1982 (IQR \$1281-\$3365)) compared with patients who were HF-non-ACHDs (\$1964 (IQR \$1216$\$ 3314)$ ); OR $1.04,95 \%$ CI 1.02 to $1.07, \mathrm{p}<0.001)$. There was an increase in ED visits across both patients with ACHD and patients who were HF-non-ACHDs that were not significantly different during the 10 -year study period (online supplemental figure 1).

\section{ACHD-related HF care during hospitalisation}

Invasive haemodynamic assessment (cardiac catheterisation or Swann central catheter) was more often used in ACHD-HFs, although less often in SV-ACHD-HFs compared with 2V-ACHD-HFs, which may be related to decrease Swann central catheter use in SV physiology due to increased thrombosis risk with an indwelling catheter in a passive venous cavopulmonary circulation (table 2). There was increased use of advanced HF 


\begin{tabular}{|c|c|c|c|c|c|c|c|}
\hline & $\begin{array}{l}\text { HF-non-ACHD, } \\
\mathrm{N}=31137414\end{array}$ & $\begin{array}{l}\text { ACHD-HF } \\
\mathrm{N}=76557\end{array}$ & $\begin{array}{l}P \text { value (vs non- } \\
\text { ACHD) }\end{array}$ & $\begin{array}{l}\text { SV-ACHD-HF, } \\
\mathrm{N}=2775\end{array}$ & $\begin{array}{l}P \text { value (vs non- } \\
\text { ACHD) }\end{array}$ & $\begin{array}{l}\text { 2V-ACHD-HF, } \\
\mathrm{N}=73782\end{array}$ & $\begin{array}{l}P \text { value (vs } \\
\text { non-ACHD) }\end{array}$ \\
\hline Age, median (IQR) & $74(63-83)$ & $61(44-75)$ & $<0.0001$ & $33(25-44)$ & $<0.0001$ & $61(45-76)$ & $<0.0001$ \\
\hline Male, n (\%) & $15205497(49)$ & $38211(50)$ & 0.0043 & $1474(53)$ & 0.073 & $36839(50)$ & 0.011 \\
\hline White, $n(\%)$ & $22593832(72)$ & $51545(69)$ & $<0.0001$ & $1857(70)$ & $<0.0001$ & $49688(70)$ & $<0.0001$ \\
\hline Insurance, n (\%) & & & $<0.0001$ & & $<0.0001$ & & $<0.0001$ \\
\hline Medicare & $23800830(77)$ & $42276(55)$ & & $800(29)$ & & $41476(56)$ & \\
\hline Medicaid & $2407875(8)$ & $11106(14)$ & & $685(25)$ & & $10421(14)$ & \\
\hline Private & $3620714(12)$ & $18149(24)$ & & 1089 (39) & & $17061(23)$ & \\
\hline Self-pay & $669446(2)$ & $2861(4)$ & & $93(3)$ & & $2768(4)$ & \\
\hline Hospital affiliation, $\mathrm{n}(\%)$ & & & $<0.0001$ & & $<0.0001$ & & $<0.0001$ \\
\hline Teaching & $15316925(50)$ & $46592(62)$ & & $2149(78)$ & & $44442(61)$ & \\
\hline Non-teaching & $15253052(50)$ & $28479(38)$ & & $592(22)$ & & $27887(39)$ & \\
\hline \multicolumn{8}{|l|}{ Comorbidities, n (\%) } \\
\hline Arrhythmia & $14344536(46)$ & $40634(53)$ & $<0.0001$ & 1699 (61) & $<0.0001$ & $38934(53)$ & $<0.0001$ \\
\hline Pulmonary hypertension & $4035064(13)$ & $20388(27)$ & $<0.0001$ & $627(23)$ & $<0.0001$ & $19761(27)$ & $<0.0001$ \\
\hline Hypertension & $12332085(40)$ & $27041(35)$ & $<0.0001$ & $380(14)$ & $<0.0001$ & $26661(36)$ & $<0.0001$ \\
\hline Diabetes & $13421724(43)$ & $20715(27)$ & $<0.0001$ & $240(9)$ & $<0.0001$ & $20475(28)$ & $<0.0001$ \\
\hline Chronic kidney disease & 11669769 (37) & $18888(25)$ & $<0.0001$ & $591(21)$ & $<0.0001$ & $18297(25)$ & $<0.0001$ \\
\hline Obesity & $5162547(17)$ & $11896(16)$ & 0.0023 & $238(9)$ & $<0.0001$ & $11658(16)$ & 0.025 \\
\hline Smoking & $7313929(23)$ & $15844(21)$ & $<0.0001$ & $314(11)$ & $<0.0001$ & $15529(21)$ & $<0.0001$ \\
\hline COPD & $4954125(16)$ & $6719(9)$ & $<0.0001$ & $70(3)$ & $<0.0001$ & $6649(9)$ & $<0.0001$ \\
\hline Coronary artery disease & $11263517(36)$ & $23615(31)$ & $<0.0001$ & $302(11)$ & $<0.0001$ & $23313(32)$ & $<0.0001$ \\
\hline
\end{tabular}

NIS database.

ACHD-HF, adult with congenital heart disease and heart failure; COPD, chronic obstructive pulmonary disease; HF-non-ACHD, non-ACHD with HF; NIS, Nationwide Inpatient Sample; SV, single-ventricle; 2V, two-ventricle.

therapies including transplant, ECMO and IABP among patients with ACHD. Interestingly, after adjusting for patient-level and centre-level factors, 2V-ACHD-HFs were less likely to receive a VAD compared with HF-non-ACHDs. Notably, SV-ACHD-HFs required remarkably high acuity care, including heart transplantation in $1.8 \%$ of admissions (compared with $0.04 \%$ of HF-nonACHDs), VAD implant in $0.7 \%$ of admissions (compared with $0.07 \%$ of HF-non-ACHDs) and ECMO support in $0.4 \%$ of admissions (compared with $0.05 \%$ of HF-non-ACHDs).

After adjusting for patient-level and centre-level factors (including age, gender, insurance type, hospital affiliation, year and comorbidities), hospital mortality was higher among SV-ACHD-HFs (6.6\%; OR 1.61, 95\% CI 1.16 to 2.26$)$ and 2V-ACHD-HFs (6.3\%; OR 1.15, IQR 1.08-1.24) compared with HF-non-ACHD (5.5\%), with a p value of $<0.001$ for both. Among those requiring ECMO support, mortality was similarly

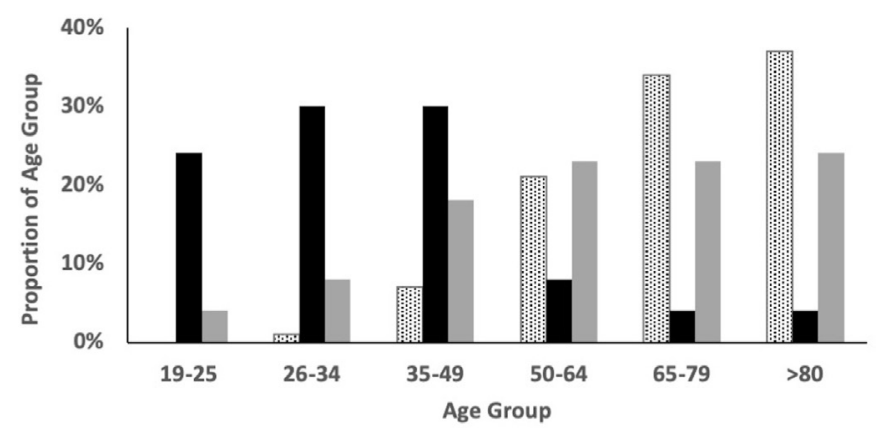

[ais HF-non-ACHD $\square$ Fontan ACHD-HF $\square$ Two-Ventricle ACHD-HF

Figure 2 Age at presentation for ACHD-HF versus HF-non-ACHD visits. ACHD-HF, adults with congenital heart disease and heart failure; HFnon-ACHD, non-ACHD with HF. high in both HF-non-ACHDs (56\%) and 2V-ACHF-HFs (54\%) ( $\mathrm{p}$ value $=0.89$ ). However, ECMO use among SV-ACHD-HF had $100 \%$ mortality. Mortality was higher in patients with ACHD who received VAD (2V-ACHD-HFs 54\% and SV-ACHD-HFs 76\%) compared HF-non-ACHDs (16\%). Similarly, mortality was higher in patients with ACHD who received transplants (2V-ACHD-HFs 11\% and SV-ACHD-HFs 30\%) versus those without ACHD (5\%).

Hospital LOS was longer for ACHD-HFs (median LOS 5 days (IQR 2-8)) and hospitalisation costs were greater (median \$42 800 (IQR \$18 400-\$106900)) compared with those without ACHD (median LOS 4 days (IQR 2-7) and median cost \$28 000 (IQR \$14 500-\$57800)), with a p value of $<0.001$ for both. Additionally, hospitalisation costs increased for all groups during the study period from 2006 to 2016, but growth was substantially higher for ACHD-HF, for whom median hospitalisation costs doubled (75\% increase) compared with HF-non-ACHD (32\% increase, $\mathrm{p}<0.001)$ (online supplemental figure 2). Hospitalisation rates also increased more among ACHD-HFs (46\%) versus HF-non-ACHD (6\%) during the study period (figure 3 and online supplemental figure 3 ). When evaluated by physiology, SV-ACHD-HFs had a $119 \%$ increase in annual hospitalisations and 2V-ACHD-HFs had a $43 \%$ increase.

However, despite the marked increase in hospitalisation for ACHD-HFs as compared with patients who were HF-nonACHDs, there was significant disparity in advanced HF support across groups (figure 3). Notably, VAD support increased by $294 \%$ in hospitalised HF-non-ACHDs (from $0.02 \%$ to $0.1 \%$ ) but remained unchanged in those with ACHD (from $0.34 \%$ to $0.33 \%$, p value $<0.001$ ). Similarly, the proportion of hospitalised patients with HF who underwent heart transplantation increased by $73 \%$ (from $0.01 \%$ to $0.05 \%$ ) for HF-non-ACHDs 


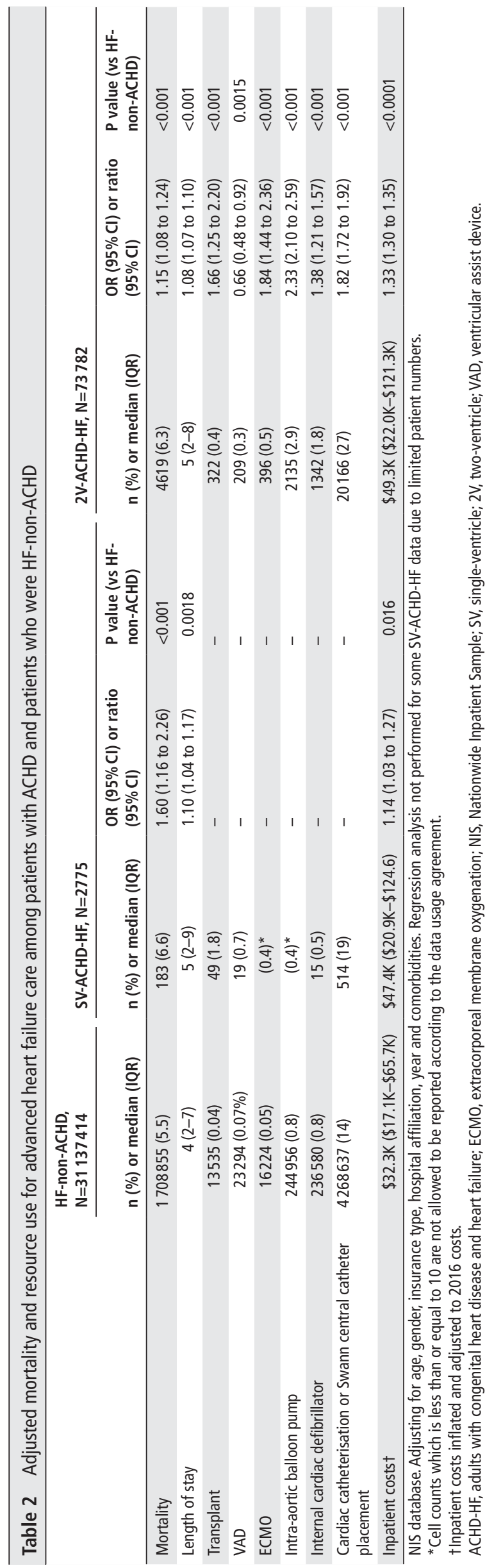

but decreased by $37 \%$ among ACHD-HFs (from $0.6 \%$ to $0.38 \%$ ) ( $\mathrm{p}$ value $<0.001$ ).

\section{DISCUSSION}

With increased survival among patients with ACHD and median age of death approaching that of the general population, the prevalence of HF in ACHD is increasing, resulting in a growing, resource-intense population. Among patients with ACHD, HF is the leading cause of death. ${ }^{6}$ In this large contemporary nationwide study of ED and inpatient hospital care in adults with HF who lack CHD as compared with ACHD-HFs, there are notable differences in both outcomes and resource use. Consistent with prior studies from earlier time periods, our data demonstrate ongoing growth in hospital resource use for patients who are ACHD-HF. ${ }^{15}$ However, our study is the first to evaluate ED-related care among patients who are ACHD-HFs. We demonstrate an increasing number of ACHD-HF ED visits similar to HF-non-ACHD over the study period, but more patients who were ACHD-HFs (78\%) required hospital admission than HF-non-ACHDs (70\%). Although ED mortality was low overall, the mortality of patients who were SV-ACHD-HFs was twice that of 2V-ACHD-HF and HF-non-ACHD. This highlights the need for early recognition of HF symptoms in adults with SV physiology who often require high-acuity care when presenting to the ED.

In-hospital mortality was significantly higher in ACHD-HFs (6.3\%), particularly SV-ACHD-HFs (6.8\%), compared with HF-non-ACHD (5.7\%), despite being younger and with fewer comorbidities. Notably, the mortality from our study is lower than previously published in the Dutch CONCOR registry that reported $4 \%$ and $35 \%$ mortality rates of 1 and 3 years, respectively, among ACHD-HFs. ${ }^{13}$ This difference may relate to inclusion of posthospital discharge mortality data in the Dutch registry, which is different from our study that was limited to in-hospital mortality. Because we excluded patients with lower-complexity CHD, our findings may underestimate the true burden of HF in ACHD, as recent data demonstrate a high burden of cardiovascular morbidity, including HF, in such patients. ${ }^{8}$ Notably, among patients with ACHD who required advanced HF therapies, mortality was significant and illustrates the high complexity of this challenging population.

Over the past decade, a growing number of patients who were ACHD-HFs have presented to the ED and required hospitalisations. This rate of growth currently exceeds those in the population that is HF-non-ACHD. Although patients who are ACHD-HFs comprise only a small portion of the overall adult HF population, their complex anatomy requires high acuity care and significant resource use, with 5-10 times higher advanced HF therapy use, including VAD, heart transplant and ECMO. Despite the rise in ACHD-HF hospitalisations, there has not been a comparable increase in advanced HF therapies, including VAD or transplantation, among patients who are ACHD-HF, highlighting a disparity in advanced HF treatment between those with and without congenital anomalies. Specifically, in our study, despite the rising incidence of hospitalisation among patients who were ACHD-HF patients, there was a decreased proportion of patients who were ACHD-HFs receiving heart transplantation. Additionally, use of VAD support remained relatively unchanged during the 10-year study period, compared with nearly a 300\% increase in VAD use among adults without CHD. Consistent with these data, Dimopoulos et al also found the number of transplants among ACHD did not parallel the expansion of the ACHD population in a UK population. ${ }^{22}$ It 


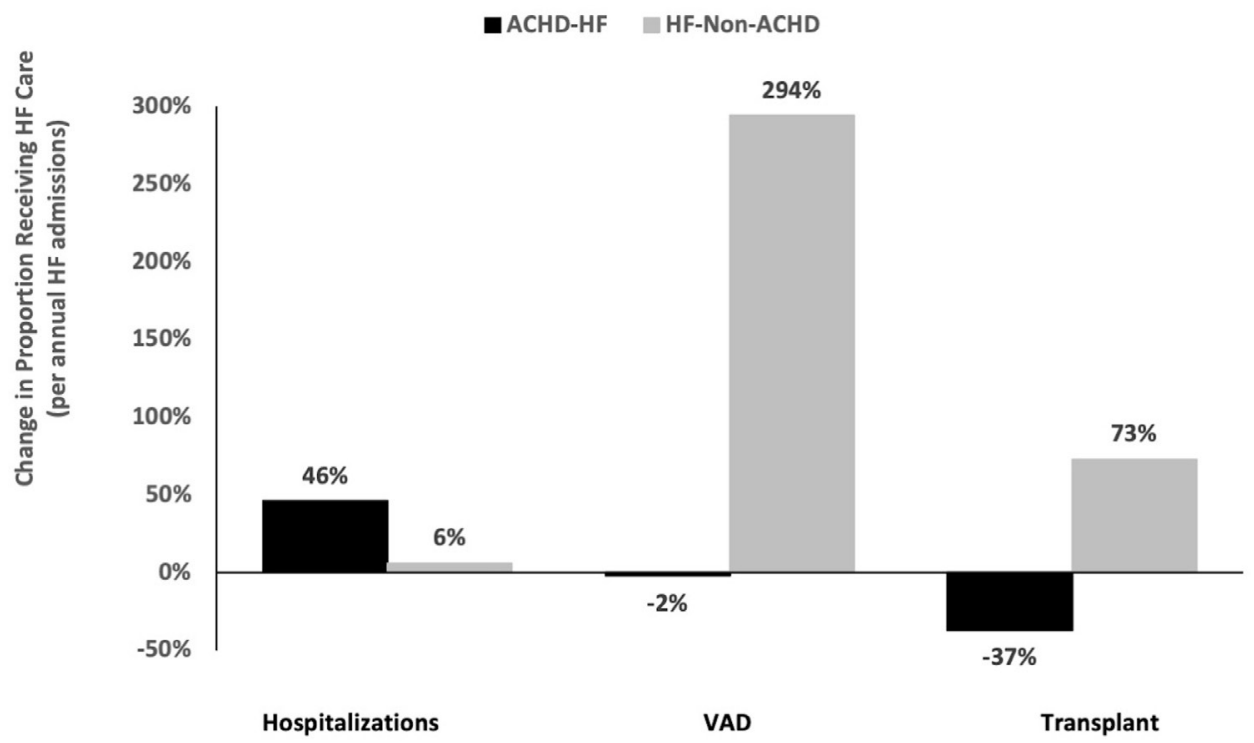

Figure 3 Change in HF resource use from 2006 to 2016 among ACHD-HF and HF-non-ACHD. ACHD-HF, adults with congenital heart disease and heart failure; VAD, ventricular assist device.

appears that complex CHD anatomy has underuse of advanced HF therapies, including VAD and heart transplantation, despite data that patients who are ACHD-HFs can be successfully treated with VAD and heart transplant with encouraging longterm outcomes. ${ }^{16}{ }^{23}$ These data suggest a need for increased advanced HF therapy consideration in patients with ACHD to improve outcomes in this high-risk population.

This study also demonstrates significantly more hospital resource use, including longer LOS and greater hospitalisation costs for ACHD-HF, but lower rates of VAD implantation and heart transplantation in such patients. Patients who are ACHD-HFs present management challenges due to their diverse presentation and complex anatomy and haemodynamics. A multidisciplinary team with expertise in the care of patients with ACHD with HF may improve outcomes, improve efficiency and decrease cost. ${ }^{64-26}$ Additionally, patients who are ACHD-HFs require significant hospital resource use near the end of life; there may be an opportunity to improve care through the incorporation of palliative and supportive care. ${ }^{27}$ Understanding these data is important for considering allocation of resources within the larger adult HF population, including use of advanced HF therapies such as transplantation and VAD.

\section{Limitations}

This study has several limitations related to its retrospective nature. First, this study is subject to the limitations inherent to an administrative database, although HCUP performs quality analyses to ensure internal consistency and data validity. Second, because these data were based on admission-level instead of patient-level data, there were limitations regarding data of patients with recurrent admissions. There may be some misclassification error due to use of ICD-9 and ICD-10 codes for identifying the study cohort, although ICD coding has been used by others as a reliable method to identify CHD-related mortality in the USA. ${ }^{28-30}$ Despite these limitations, these data remain important to present and understand as they provide a comprehensive analysis of both ED and hospital care for patients who are ACHD-HFs in the current era.

\section{CONCLUSION}

ACHD-HF is a growing population with care that is associated with high ED and hospital resource use. Despite increased ED visits and hospitalisations, there remains a disparity in use of advanced HF therapies, including VAD and transplantation, when compared with adults with HF but without ACHD. Focusing on care for ACHD-HFs care in specialised centres may improve outcomes and resource use in this complex population.

\section{Key messages}

What is already known about this subject?

- Among adults with congenital heart disease, heart failure (HF) is the leading cause of mortality. However, limited contemporary data exist regarding heart failure (HF) outcomes and resource use in adult congenital heart disease (ACHD).

What might this study add?

- This study aimed to understand differences in outcomes and resource use among adults with $\mathrm{HF}$ and $\mathrm{ACHD}$ compared to those without $A C H D$ in the modern era.

- In this large contemporary cohort, adults with congenital heart disease and heart failure (ACHD-HFs) had higher mortality ( $6.3 \%$ vs $5.5 \%)$, higher ED admissions ( $78 \%$ vs $70 \%$ ), longer length of stay (5 days vs 4 days) and greater hospital costs (\$49K vs \$32K) compared to non-ACHD with HF (HF-non-ACHDs).

- Despite a greater increase in HF hospitalisations over the 10-year period among those with ACHD (46\%) compared to those without ACHD (6\%), the proportion of ACHD-HFs receiving $\operatorname{VAD}(-2 \%)$ or transplant $(-37 \%)$ decreased.

How might this impact clinical practice?

- Despite increasing hospital resource use among adults with ACHD and HF, advanced HF therapies are used less often compared to adults without CHD, highlighting potential utility of specialised ACHD and HF centres to improve outcomes. 
Contributors DSB conceptualised and designed the study, drafted the initial manuscript, and reviewed and revised the manuscript. HG and XZ designed the data collection instruments, collected the data, carried out the initial analysis, and reviewed and revised the manuscript. JR, BF, YK, JL, JAM, JE and RF reviewed and interpreted the data analysis, and reviewed and revised the manuscript. JNM conceptualised and designed the study, coordinated and supervised data collection, and critically reviewed the manuscript for important intellectual content. All authors approved the final manuscript as submitted and agreed to be accountable for all aspects of the work. The corresponding author has the right to grant on behalf of all authors and does grant on behalf of all authors, an exclusive licence on a worldwide basis to the BMJ Publishing Group Ltd and its licensees to permit this article (if accepted) to be published in HEART editions and any other BMJPGL products to exploit all subsidiary rights.

Funding This work is supported in part by the Cardiac Center Clinical Research Core at the Children's Hospital of Philadelphia. No extramural funding was used to support this work.

Competing interests None declared.

Patient consent for publication Not required.

Ethics approval The institutional review board of the Children's Hospital of Philadelphia deemed this study exempt from review under 45 CFR 46.102(f), as patient data were deidentified.

Provenance and peer review Not commissioned; externally peer reviewed.

Data availability statement All data relevant to the study are included in the article or uploaded as supplementary information.

Supplemental material This content has been supplied by the author(s). It has not been vetted by BMJ Publishing Group Limited (BMJ) and may not have been peer-reviewed. Any opinions or recommendations discussed are solely those of the author(s) and are not endorsed by BMJ. BMJ disclaims all liability and responsibility arising from any reliance placed on the content. Where the content includes any translated material, BMJ does not warrant the accuracy and reliability of the translations (including but not limited to local regulations, clinical guidelines, terminology, drug names and drug dosages), and is not responsible for any error and/or omissions arising from translation and adaptation or otherwise.

\section{ORCID iDs}

Danielle S Burstein http://orcid.org/0000-0002-9870-7607

Yuli Y Kim http://orcid.org/0000-0002-2342-192X

\section{REFERENCES}

1 Marelli AJ, Mackie AS, Ionescu-Ittu R, et al. Congenital heart disease in the general population: changing prevalence and age distribution. Circulation 2007;115:163-72.

2 Khairy P, lonescu-Ittu R, Mackie AS, et al. Changing mortality in congenital heart disease. J Am Coll Cardiol 2010;56:1149-57.

3 Gilboa SM, Devine OJ, Kucik JE, et al. Congenital heart defects in the United States: estimating the magnitude of the affected population in 2010. Circulation 2016;134:101-9.

4 Opotowsky AR, Siddigi OK, Webb GD. Trends in hospitalizations for adults with congenital heart disease in the U.S. J Am Coll Cardiol 2009;54:460-7.

5 Stout KK, Broberg CS, Book WM, et al. Chronic heart failure in congenital heart disease: a scientific statement from the American heart association. Circulation 2016;133:770-801.

6 Menachem JN, Schlendorf KH, Mazurek JA, et al. Advanced Heart Failure in Adults With Congenital Heart Disease. JACC Heart Fail 2020;8:87-99.

7 Norozi K, Wessel A, Alpers V, et al. Incidence and risk distribution of heart failure in adolescents and adults with congenital heart disease after cardiac surgery. Am J Cardiol 2006:97:1238-43.
8 Saha P, Potiny P, Rigdon J, et al. Substantial cardiovascular morbidity in adults with Lower-Complexity congenital heart disease. Circulation 2019;139:1889-99.

9 Verheugt CL, Uiterwaal CSPM, van der Velde ET, et al. The emerging burden of hospital admissions of adults with congenital heart disease. Heart 2010;96:872-8.

10 Van De Bruaene A, Hickey EJ, Kovacs AH, et al. Phenotype, management and predictors of outcome in a large cohort of adult congenital heart disease patients with heart failure. Int J Cardiol 2018;252:80-7.

11 Rodriguez FH, Moodie DS, Parekh DR, et al. Outcomes of heart failure-related hospitalization in adults with congenital heart disease in the United States. Congenit Heart Dis 2013:8:513-9.

12 Lal S, Kotchetkova I, Cao J, et al. Heart failure admissions and poor subsequent outcomes in adults with congenital heart disease. Eur I Heart Fail 2018;20:812-5.

13 Zomer AC, Vaartjes I, van der Velde ET, et al. Heart failure admissions in adults with congenital heart disease; risk factors and prognosis. Int I Cardiol 2013; 168:2487-93.

14 Moussa NB, Karsenty C, Pontnau F, et al. Characteristics and outcomes of heart failure-related hospitalization in adults with congenital heart disease. Arch Cardiovasc Dis 2017;110:283-91.

15 Burchill LJ, Gao L, Kovacs AH, et al. Hospitalization trends and health resource use for adult congenital heart disease-related heart failure. J Am Heart Assoc 2018;7:e008775.

16 VanderPluym CJ, Cedars A, Eghtesady P, et al. Outcomes following implantation of mechanical circulatory support in adults with congenital heart disease: an analysis of the Interagency Registry for mechanically assisted circulatory support (INTERMACS). J Heart Lung Transplant 2018;37:89-99.

17 Healthcare Cost and Utilization Project. Calculating National Inpatient Sample (NIS) Variances for Data Years 2012 and Later. Report \#2015-09 2014.

18 HCUP Nationwide Emergency Department Sample (NEDS). Healthcare cost and utilization project (HCUP) 2018.

19 Healthcare Cost and Utilization Project. Introduction to the HCUP nationwide emergency department sample (NEDS) 2014.

20 Feudtner C, Feinstein JA, Zhong W, et al. Pediatric complex chronic conditions classification system version 2: updated for ICD-10 and complex medical technology dependence and transplantation. BMC Pediatr 2014;14:199.

21 Us Bureau of labor statistics. consumer price index 2018.

22 Dimopoulos K, Muthiah K, Alonso-Gonzalez R, et al. Heart or heart-lung transplantation for patients with congenital heart disease in England. Heart 2019;105:596-602.

23 Burchill $\amalg$, Edwards LB, Dipchand Al, et al. Impact of adult congenital heart disease on survival and mortality after heart transplantation. J Heart Lung Transplant 2014;33:1157-63.

24 Sabanayagam A, Cavus O, Williams J, et al. Management of heart failure in adult congenital heart disease. Heart Fail Clin 2018;14:569-77.

25 Alshawabkeh LI, Opotowsky AR. Burden of heart failure in adults with congenital heart disease. Curr Heart Fail Rep 2016;13:247-54.

26 Menachem JN, Golbus JR, Molina M, et al. Successful cardiac transplantation outcomes in patients with adult congenital heart disease. Heart 2017;103:1449-54.

27 Steiner JM, Kirkpatrick JN, Heckbert SR, et al. Hospital resource utilization and presence of advance directives at the end of life for adults with congenital heart disease. Congenit Heart Dis 2018;13:721-7.

28 Boneva RS, Botto LD, Moore CA, et al. Mortality associated with congenital heart defects in the United States: trends and racial disparities, 1979-1997. Circulation 2001;103:2376-81.

29 Burstein DS, Shamszad P, Dai D, et al. Significant mortality, morbidity and resource utilization associated with advanced heart failure in congenital heart disease in children and young adults. Am Heart J 2019;209:9-19.

30 Edelson JB, Rossano JW, Griffis H, et al. Emergency department visits by children with congenital heart disease. J Am Coll Cardiol 2018;72:1817-25. 